\title{
Research of the spectral diffuse reflectance of melanoma in vivo
}

\author{
V.G. Petruk ${ }^{* a}$, D.B. Bolyuh ${ }^{\mathrm{b}}$, S.M. Kvaternyuk ${ }^{\mathrm{a}}$, O.E. Kvaternyuk ${ }^{\mathrm{c}}$, Y.M. Denysiuk $^{\mathrm{a}}$, A. Kotyra $^{\mathrm{d}}$ \\ ${ }^{a}$ Vinnytsia National Technical University, Khmelnytske Shose, 95, Vinnitsa, 21021, Ukraine; \\ ${ }^{b}$ Vinnytsia National Medical University, str. Pirogov 56, Vinnytsia, 21018, Ukraine; \\ ${ }^{\mathrm{c}}$ Vinnytsia Humanitarian Pedagogical College, Vinnytsia, Ukraine; \\ ${ }^{\mathrm{d}}$ Lublin University of Technology, Nadbystrzycka 38A, 20-618 Lublin, Poland;
}

\begin{abstract}
The structure and principle of operation of information-measuring system of diagnostics of normal and pathological biological tissues from the spectrum of diffuse reflection. Research of spectrum of diffuse reflection of patients with melanoma and comparison of characteristics of normal skin relatively healthy recipient. The measurement results can be used by an expert system based on fuzzy logic for decision support physician about diagnosis and correction methods of treatment.
\end{abstract}

Keywords: biological tissues, melanoma, spectrophotometry, diagnostics.

\section{INTRODUCTION}

Biomedical diagnosis of melanoma is an important opportunity for non-invasive research, because even slight damage malignancies may be the impetus for their regeneration and rapid growth. Among the methods that can provide it, stipulates optical, including spectrophotometric. Improved methods of diagnosis of normal and pathological tissues by diffuse reflectance spectra of them can also be used in other areas of medicine - diagnosis of patients with amputated limbs, forensic and so on.

The object of study is the measurement process by means of information-measuring system diffuse reflectance spectrum of skin to pathological tissues of patients with melanoma and compared them with properties for conventionally healthy recipients. Purpose - a theoretical feasibility study of new methods of diagnosing melanoma patients by diffuse reflectance spectra, development of basic principles of diagnosis and their experimental verification, development of special expertise and techniques of diagnosis non-invasive surface characteristics of tissues.

\section{THE STRUCTURE AND PRINCIPLE OF OPERATION OF INFORMATION- MEASURING SYSTEM}

The device consists of a source of monochromatic radiation monochromator a wavelength which changes stepper motor, two photometric probes 2 and 3 with identical parameters. As an initial optical transducer used integrating sphere by the method of Taylor, which is most appropriate for non-invasive measurements. Each of photometric probes should integrate a spherical cavity, covered within exemplary optically-reflective substance, such as magnesium oxide.

\footnotetext{
*petrukvg@gmail.com, phone: +380 (432) 59-84-43
}

Optical Fibers and Their Applications 2012, edited by Ryszard S. Romaniuk, Waldemar Wójcik, Proc. of SPIE Vol. 8698, 86980F · (C) 2012 SPIE · CCC code: 0277-786/12/\$18 · doi: 10.1117/12.2019730 
Entrance hole probes 2 and 3 are connected to a source of monochromatic radiation of a fiber-optical fiber 4 and 5 . Working holes photometric probes are on the same axis with the inlet and outlets located in them with radiation detectors 6 and 7 are perpendicular to the common axis of the input and job openings. Axles outlets overlap with common axes of input and running holes in the center of a spherical cavity. In ports photometric probes installed prefabricated quartz lens 8 and 9 of the optical parameters that provide the same diameter of the beam in your hole, as the diameter of the working aperture. The design tool also includes plugs 10 for job openings photometric probes. The surface of the plug is covered with the same perfectly-reflective substance, as well as integrating spherical cavity of the same photometric probes.

Photodetectors electrically connected with a registering apparatus, which can be used as a separate unit 11 . The structure also includes a microcontroller means 12 for the processing of information, which is connected to the registration apparatus 11 and a source of monochromatic radiation, 1 and 13 PC with specialized software 14 for processing the spectra of diffuse reflection and problem-oriented expert system 15 decision decisions connected to the microcontroller 12.

The proposed tool works as follows. Beams of monochromatic radiation of a wavelength, the source formed by a fiberoptic light 4 and 5 and the lens 8 and 9 are aimed at working apertures photometric probes 2 and 3 . In the case of measuring the skin diagnosis (normal or abnormal) working aperture of a photometric probes, such probes 2, closing cap 10 . Working aperture photometric probes 3 tightly pressed against the working aperture to the study of pathological areas of the skin.

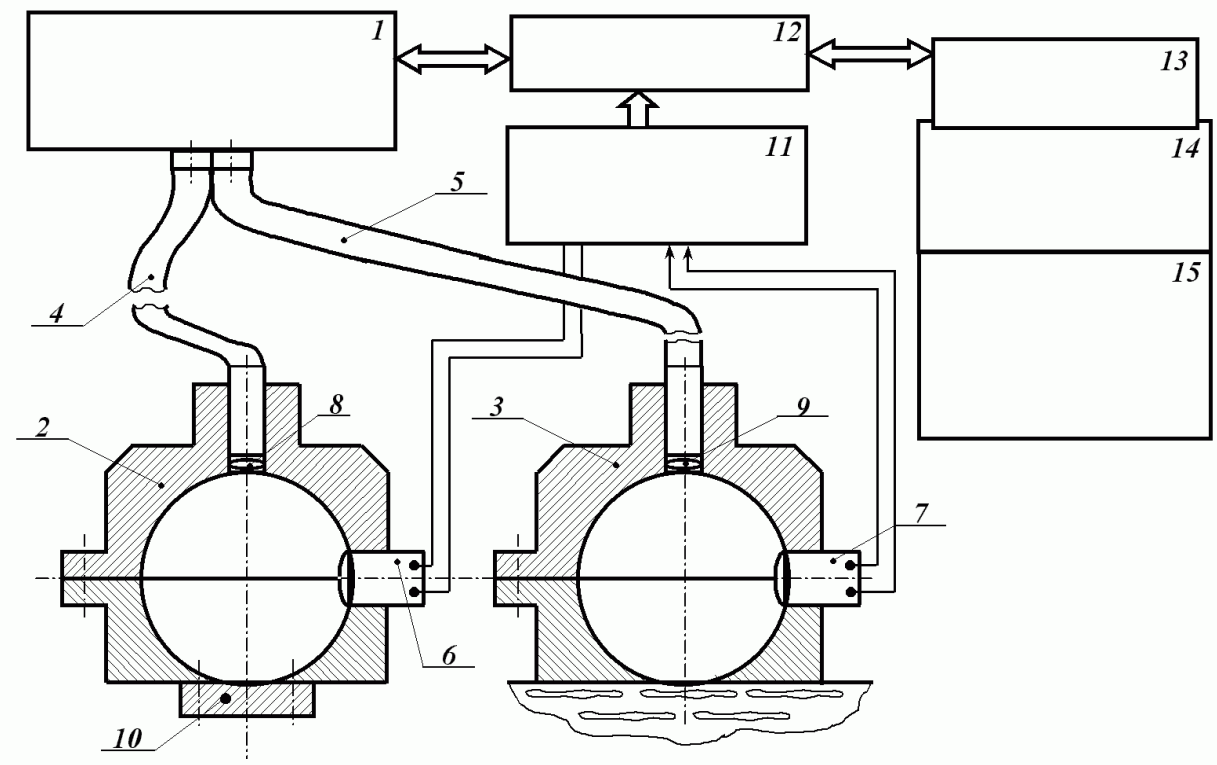

Figure 1. Information-measuring system for investigation of diffuse reflection tissues.

Beams of light diffusely reflected from objects of study are integrated in spherical primary converters, making them a spatial brightness whose elements are perceived photodetector 6 and 7, causing their outputs corresponding information signals. These signals are amplified in block registration devices 10 and submitted for comparison to the bridge circuit microcontroller 12 then coded and processed by personal computer 13. Treatment of diffuse reflectance spectrum and their comparison with the norm is using specialized software 14, and set the type of pathology by a computer problemoriented expert system 15 , based, for example, fuzzy $\operatorname{logic^{1,2}}$. 


\section{ANALYSIS OF THE DIFFUSE REFLECTANCE SPECTRUM OF THE DIAGNOSIS OF MELANOMA}

Diffuse reflectance spectrum of normal and pathological tissues was performed in the range from 560 to $1050 \mathrm{~nm}$, which limited the spectral sensitivity characteristics of silicon photodiodes used in this information-measuring systems as radiation detectors 6,7 . Although a monochromator provides spectral range from 200 to $1200 \mathrm{~nm}$, on the borders of this range of information signals from photodiodes decreases and approaches the level of noise. Diffuse reflectance spectrum in the range from 560 to $1050 \mathrm{~nm}$ allows to diagnose the characteristics of melanoma, because this range is the range of characteristic wavelengths specific for the disease pigments, primarily melanin. Each wavelength is 1000 measurements and averaging them, which significantly reduced the random errors caused by noise signals.

At the beginning of the compensation is dark currents of photodiodes and normalization of the spectral characteristics of the measuring channels relative measurement model vehicle. To do this, both photometric probes close cap covered with perfectly-reflective substance and held a series of measurements. Information-measuring system records the signal from a measuring probe against the other. The resulting characteristics (D0 in Figure 2) can compensate for differences between the spectral sensitivity of radiation detectors for each channel. Then cover with working photometric probe is removed and held non-invasive in vivo measurement of diffuse reflectance spectrum of pathological skin melanoma patients (D1), clean intact skin melanoma patients (D2), normal skin relatively healthy recipient (D3) and melanoma specimen obtained after surgical removal (D4). Spectral characteristics of the original raw data to information-measuring system shown in Figure 2.

The measured spectrum of diffuse reflection tissues are entered in a database with the date of measurement, names, age and type of disease the patient, a number of additional medical characteristics obtained by other tests for comparison of their spectral data.

The coefficient of diffuse reflection of the skin get after correcting the measured values of $\mathrm{D}_{\mathrm{i}}$ a factor that takes into account the differences between the spectral sensitivity of measuring channels $\mathrm{D}_{0}: k_{D R i}=D_{0} / D_{i}$. Comparison of various diagnostic facilities relative change in the ratio of diffuse reflectance in the spectral range of easy to make on the basis of normalized values of diffuse reflectance relative to their maximum value: $k_{D R i}^{\prime}=k_{D R i} / \max \left(k_{D R i}\right)$.

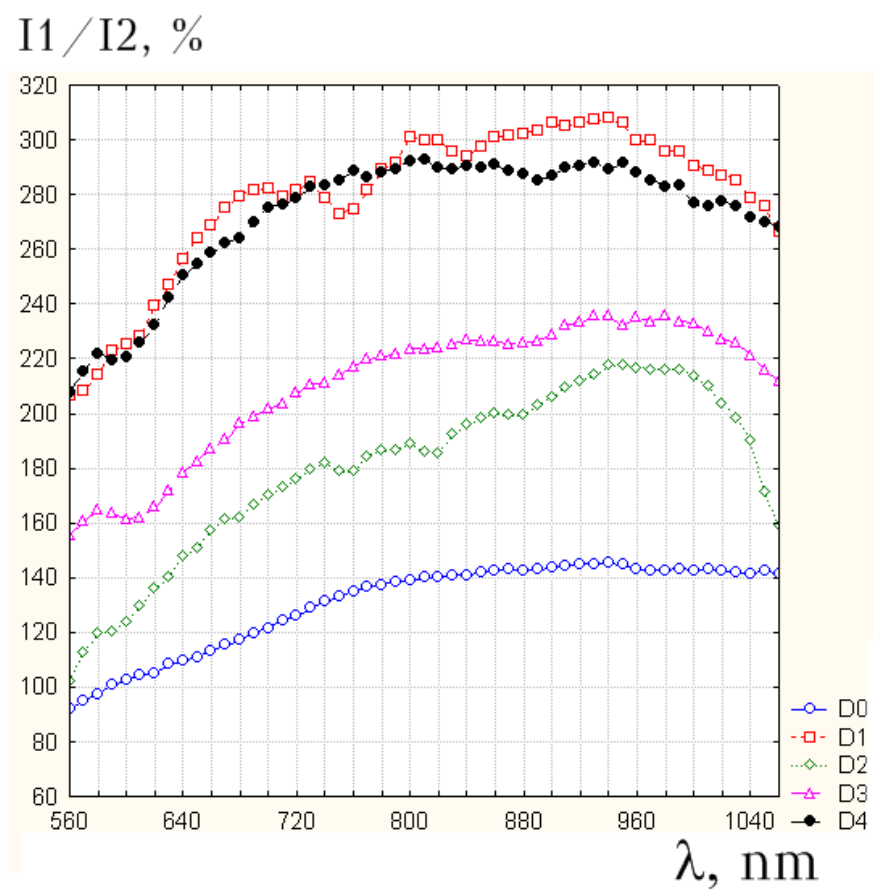

Figure 2. Spectral characteristics of the reflection parameter of normal and pathological tissues. 
A comparison of the spectral coefficients of diffuse reflection of the skin $\mathrm{k}_{\mathrm{DR}}$ and normalized values of $\mathrm{k}_{\mathrm{DR}}$ for the following cases:

- $\quad$ to clean intact skin melanoma patients and normal skin relatively healthy recipients (Figure 3);

- $\quad$ skin melanoma patients and clean intact skin melanoma patients (Figure 4);

- $\quad$ skin melanoma patients and melanoma specimen obtained after surgical removal (Figure 5);

- conditionally normal skin of healthy recipient and melanoma sample obtained after surgical removal (Figure 6).

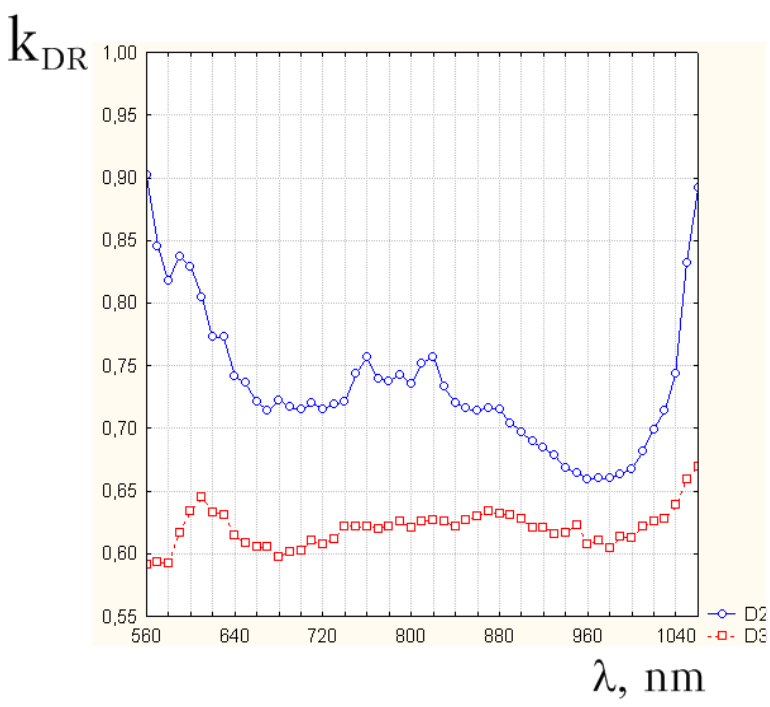

(a)

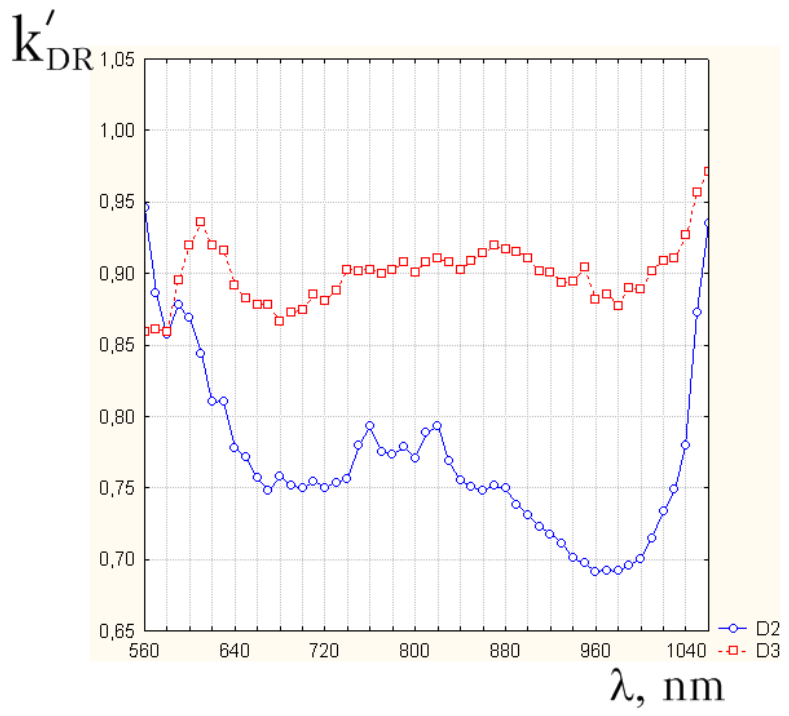

(b)

Figure 3. The coefficients of diffuse reflection $\mathrm{k}_{\mathrm{DR}}$ (a) and $\mathrm{k}_{\mathrm{DR}}$ (b) for pure intact skin melanoma patients (D2) and normal skin relatively healthy recipient (D3).

Analyze the results. In figure 3 the coefficient of diffuse reflection $\mathrm{k}_{\mathrm{DR}}$ conditionally normal skin of healthy recipient for 6-15\% more than in the case of clean intact skin melanoma patients (the largest difference at a wavelength of $600 \mathrm{~nm}$ ). When comparing the normalized values of $\mathrm{k}^{\prime}{ }_{\mathrm{DR}}$ difference is $20 \%$ (the largest difference at the wavelength $960-980 \mathrm{~nm}$ ), allowing confident diagnosis of melanoma. It should be noted that in this case the comparison was carried out on patients with melanoma stage 4 disease. The difference for patients in stage 1 and 2 may not be so great.

In Figure 4 coefficient of diffuse reflection kDR for pathological skin melanoma patients to $18-25 \%$ less than in the case of pure intact skin melanoma patients. When comparing the normalized values of $\mathrm{k}^{\prime}{ }_{\mathrm{DR}}$ difference is $20 \%$. 


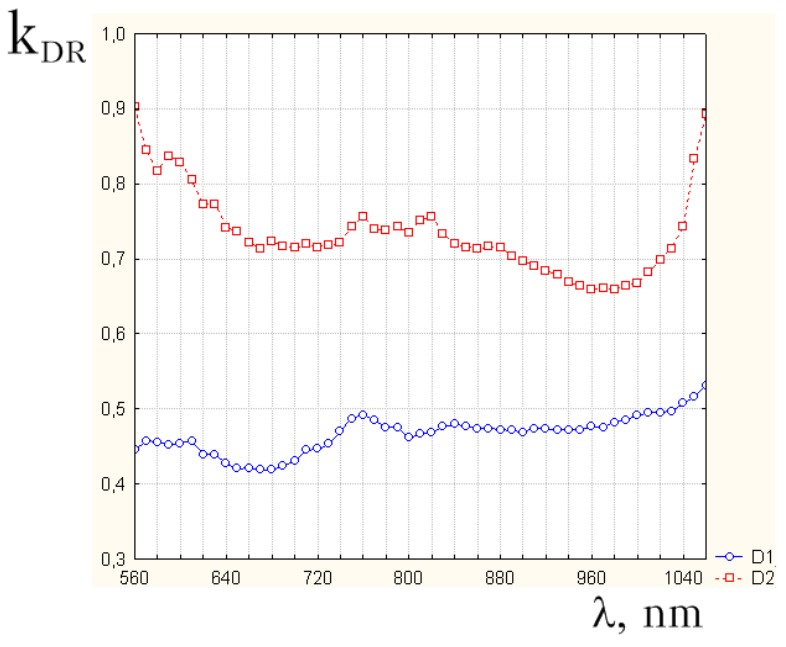

(a)

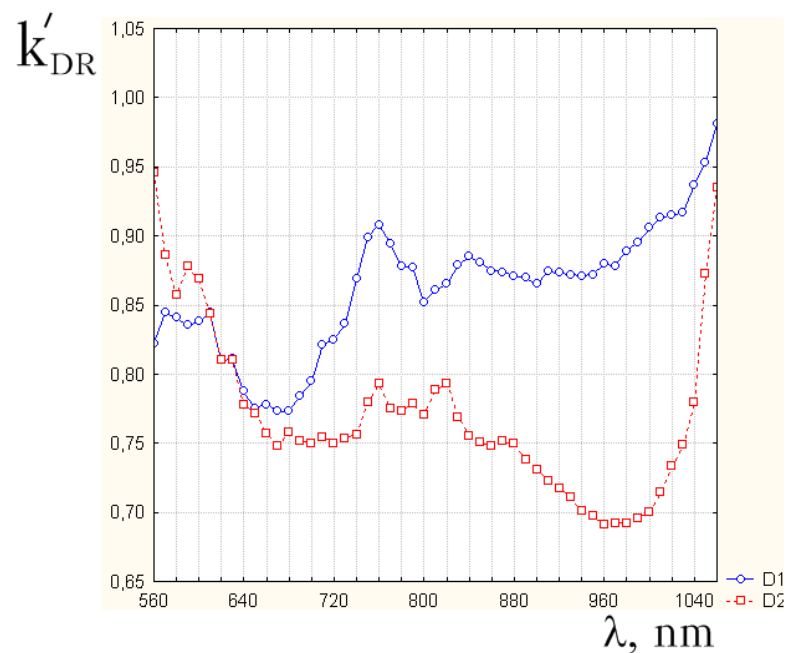

(b)

Figure 4. The coefficients of diffuse reflection $k_{D R}(a)$ and $k^{\prime}{ }_{D R}(b)$ for for pathological skin melanoma patients (D1) and clean intact skin melanoma patients (D2).

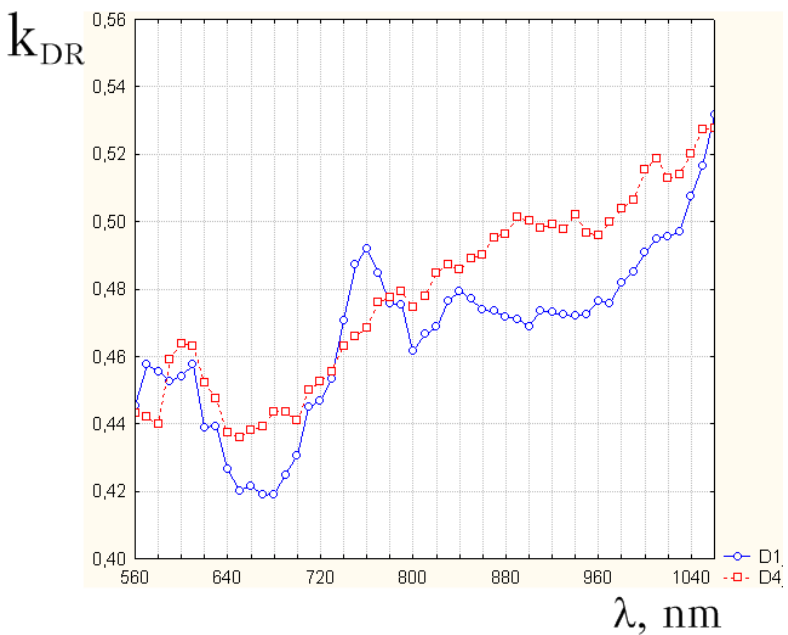

(a)

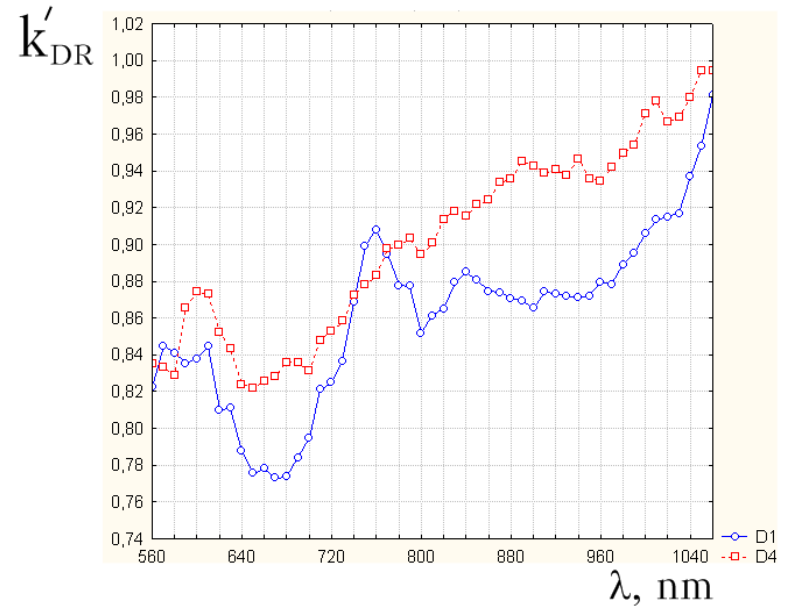

(b)

Figure 5. The coefficients of diffuse reflection $\mathrm{k}_{\mathrm{DR}}$ (a) and $\mathrm{k}_{\mathrm{DR}}(\mathrm{b})$ for pathological skin melanoma patients (D1) and melanoma specimen obtained after surgical removal (D4).

In figure 5 the coefficient of diffuse reflection $\mathrm{k}_{\mathrm{DR}}$ for pathological skin melanoma patients and melanoma specimen obtained after surgical removal differ by $2-3 \%$. Some deviations may be caused by lack of blood supply and gradual temporal changes in the tumor. 


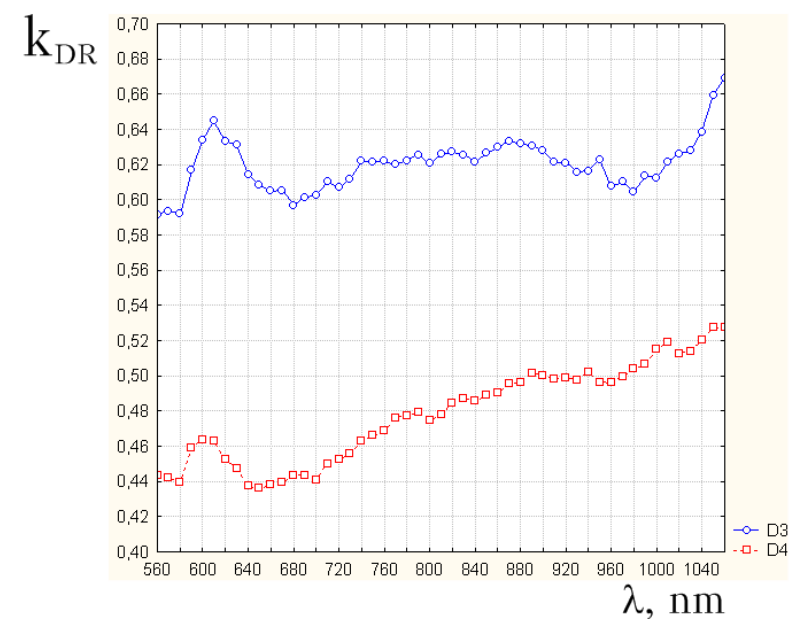

(a)

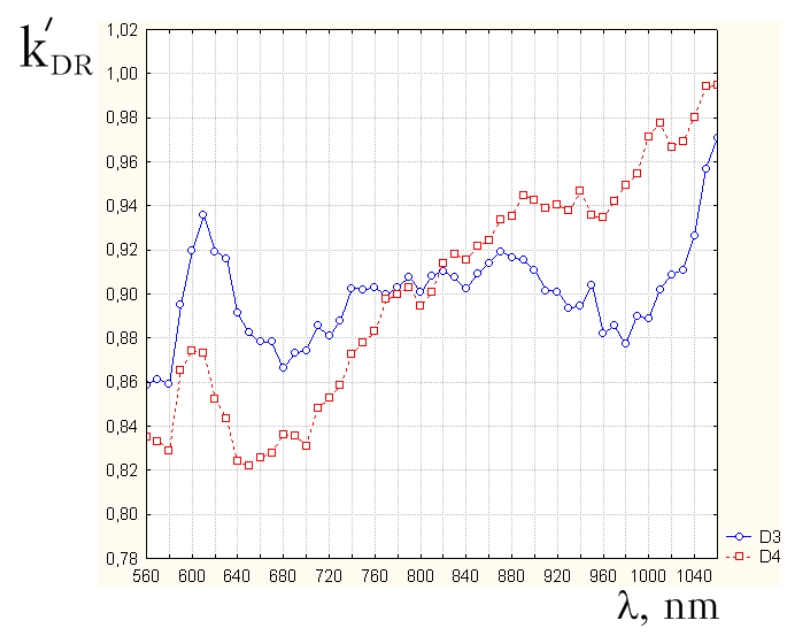

(b)

Figure 6. The coefficients of diffuse reflection $\mathrm{k}_{\mathrm{DR}}(\mathrm{a})$ and $\mathrm{k}_{\mathrm{DR}}(\mathrm{b})$ for normal skin relatively healthy recipient (D3) and melanoma specimen obtained after surgical removal (D4).

In Figure 6 the coefficient of diffuse reflection $k_{\mathrm{DR}}$ normal skin relatively healthy recipients by $10-15 \%$ greater than for melanoma sample obtained after surgical removal.

\section{CONCLUSIONS AND RECOMMENDATIONS}

The results of measurements of diffuse reflectance spectrum can be used by an expert system that is based on fuzzy logic for decision support doctor about diagnosis and correction methods of treatment.

Further research and accumulate data bank diffuse reflectance spectra that allow the expert system are more likely to make correct diagnosis.

Research carried out by scientific teams of the Department of Ecology and Environmental Safety Vinnytsia National Technical University and the department of oncology, radiation diagnostics and radiation therapy Ukraine National Pirogov Memorial Medical University, Vinnitsa.

\section{REFERENCES}

[1] Petruk, V.G., Chernovolyk, G.A., Vasilkovsky, I.V. and Tomchuk, M.A., "Device for non-invasive optical diagnostic biomedical materials," Ukraine Patent 46340A, May 15, 2002.

[2] Petruk, V.G., Kvaternyuk, S.M., Chernovolyk, G.A., Vasilkovsky, I.V., Ishchenko, V.A. and Petruk, R.V. "Evolving principles of diagnosis of normal and pathological tissues by diffuse reflectance spectra (theoretical and practical research)," Priorities and scientific cooperation SFFR-BRFFR: Proceedings of joint competitive projects, 35-47 (2007). 